\title{
Protocolo de assistência do serviço de condicionamento físico para indivíduos com doenças neuromusculares e musculoesqueléticas
}

\section{Assistance protocol of physical fitness departments for individuals with neuromuscular and musculoskeletal diseases}

Cristiane Vieira Cardoso ${ }^{1}$, Cristiane Gonçalves Mota ${ }^{1}$, Isabel Cristina da Silva Prado Sampaio ${ }^{1}$, Livia Maria dos Santos Sabbag ${ }^{2}$

\section{RESUMO}

O condicionamento físico supervisionado por profissionais de Educação Física inserido em um programa de reabilitação para pacientes com distúrbios neuromusculares e musculoesqueléticos visa o aumento da força muscular, melhora da coordenação motora, equilíbrio dinâmico, capacidade aeróbia, capacidade funcional e da aptidão física voltada à saúde. Trata-se de importante intervenção para prevenir e atenuar a sinergia entre a perda de função física e a exacerbação de várias comorbidades que comprometem a independência funcional, modificar fatores de risco, aumentar a qualidade de vida e longevidade com redução da mortalidade. O objetivo desse artigo é apresentar o protocolo de assistência do Serviço de Condicionamento Físico do Instituto de Medicina Física e Reabilitação do Hospital das Clinicas da Faculdade de Medicina da Universidade de São Paulo (IMREA-HCFMUSP), elaborado para que a intervenção de exercícios físicos seja adequada, respeitando o perfil e limitações físicas desses pacientes, bem como na observação de seus marcadores clínicos.

Palavras-chave: Doenças Neuromusculares, Doenças Musculoesqueléticas, Aptidão Física, Exercício

\begin{abstract}
Physical fitness supervised by Physical Education professionals inserted in a rehabilitation program for patients with neuromuscular and musculoskeletal disorders aims to increase muscle strength, improve motor coordination, dynamic balance, aerobic capacity, functional capacity and healthrelated physical fitness. This is an important intervention to prevent and mitigate the synergy between loss of physical function and exacerbation of various comorbidities that compromise functional independence, modify risk factors, increase quality of life and longevity with reduced mortality. The aim of this paper is to present the assistance protocol of the Physical Conditioning Service of the Institute of Physical Medicine and Rehabilitation of the Clinical Hospital of the University of São Paulo Medical School (IMREA-HCFMUSP), designed for the intervention of physical exercises, respecting the profile and physical limitations of these patients, as well as observing their clinical markers.
\end{abstract}

${ }^{1}$ Educador Físico, Instituto de Medicina Física e Reabilitação do Hospital - IMREA HCFMUSP.

${ }^{2}$ Medica Cardiologista, Instituto de Medicina Física

e Reabilitação do Hospital - IMREA HCFMUSP.

Keywords: Neuromuscular Diseases, Musculoskeletal Diseases, Physical Fitness, Exercise

\section{Correspondência: \\ Cristiane Vieira Cardoso \\ E-mail: cristiane.cardoso@hc.fm.usp.br}

Recebido em 05 Maio 2018.

Aceito em 26 Outubro 2018.

\section{Como citar}

Cardoso CV, Mota CG, Sampaio ICSP, Sabbag LMS. Protocolo de assistência do serviço de condicionamento físico para indivíduos com doenças neuromusculares e musculoesqueléticas. Acta Fisiatr. 2018;25(2):102-106. 


\section{INTRODUÇÃO}

O programa de condicionamento físico visa promover adaptações neuromusculares e musculoesqueléticas com impacto positivo sobre o controle motor associado à deficiência física. Ao atuar no processo de reabilitação física, o programa incorpora diretrizes destinadas a promover um tratamento de baixo risco, que deve ser prescrito e monitorado individualmente para que ocorra melhora da capacidade funcional dos pacientes que apresentam grandes incapacidades.

Fez-se necessária a elaboração de um protocolo de assistência para padronizar as atividades dos educadores físicos no atendimento de diferentes populações com incapacidades crônicas secundárias a doenças neuromusculares e musculoesqueléticas, com diferentes sintomas, capacidade funcional e estratégias de reabilitação. Este programa baseado em diretrizes define claramente os limites e cuidados na avaliação física e funcional, na prescrição e intervenção do treinamento aeróbio e de resistência, ampliando a abordagem das seguintes doenças: lesão encefálica, lesão da medula espinhal, amputação, doença de Parkinson, esclerose múltiplas, síndromes de Guillain Barré, e pós-poliomielite, distrofias musculares, miopatias e hemofilia.

Foi realizada uma revisão bibliográfica de artigos e livros publicados no período de 1991 a 2011, nas bases de dados científicas LILACS, Scielo e Pubmed, nos idiomas português e inglês, utilizando-se como descritores, doenças neuromusculares, condicionamento físico e utilização de livros de referência para avaliação de aptidão física voltada a saúde. Os dados foram analisados e dessa maneira, determinaram os seguintes pressupostos teóricos sobre condicionamento físico.

\section{Avaliação pré-atividade}

O programa de Condicionamento Físico para indivíduos com incapacidade crônica, contém várias etapas distintas que visam minimizar os riscos antes de o paciente ser liberado para aintervenção com exercícios, uma vez que, pacientes com grande incapacidade, podem apresentar comorbidades como insuficiência coronariana, hipertensão arterial sistêmica e diabetes mellitus entre outras. Foi adotada uma sequência lógica e prática destinada a reunir e avaliar informações de saúde a respeito do paciente. ${ }^{1}$

A primeira etapa da avaliação préatividade é a anamnese, aqui são colhidas informações pessoais do indivíduo como nome completo, grau de escolaridade, bem como histórico de saúde e exercício físico. A estratificação de risco é a segunda etapa da avalição pré-atividade, é utilizada para obter informações atuais acerca do estado atual de saúde e para classificar os pacientes em três categorias de risco, baixo, moderado e alto, esta etapa proporciona uma base para a recomendação de avaliação cardiológica, na qual todos os pacientes estratificados como de risco moderado e alto são encaminhados para avaliação médica cardiológica. ${ }^{1}$ A avaliação médica cardiológica inclui eletrocardiograma (ECG) de repouso, exames complementares se necessários, quando possível teste ergométrico ou teste de esforço cardiopulmonar para detecção de doença cardíaca, desempenho cardiorrespiratório e prescrição de treino aeróbio. Apenas após a conclusão destas etapas o paciente realiza avaliação física com o educador físico.

\section{Método de avaliação}

Na construção do método de avaliação optou-se por avaliar a aptidão física relacionada à saúde. Existem várias definições de aptidão física relacionada à saúde, no entanto a terminologia mais relevante ao público em questão é a oferecida pelo Centers for Disease Control and Prevention, "Aptidão Física é um conjunto de atributos que as pessoas possuem ou adquirem e que se relaciona com a capacidade de executar

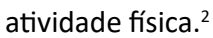

A aptidão física relacionada à saúde enfoca as capacidades funcionais relacionadas à boa saúde global e prevenção de doenças, por esta razão seus principais componentes são, aptidão cardiorrespiratória, aptidão muscular, flexibilidade da região lombo sacra e da musculatura posterior de coxa e composição corporal. $^{2}$

Existem vários testes para cada um dos quatro componentes da aptidão física relacionada à saúde, na escolha dos testes foi levada em conta, à população especifica de pacientes com grandes incapacidades motoras que frequentam este instituto, a facilidade de administração do teste e de comparação dos dados. $^{3}$

Os testes foram divididos em testes realizados em repouso; pressão arterial (PA), frequência cardíaca (FC), dextro, peso e altura e testes realizados durante o exercício; teste aptidão física e testes de capacidade funcional.

$A$ verificação de $P A, F C$ e dextro é realizada antes de cada sessão de condicionamento físico pela equipe de enfermagem, visto que os resultados interferem diretamente na tomada de decisão acerca da conveniência de realizar ou não atividade neste dia. Periodicidade das reavaliações de peso e altura e os testes realizados durante o exercício e ocorrem a cada três meses.

Dentre os muitos testes realizados em exercício para determinação da aptidão física relacionada à saúde, foram selecionados os que condizem com a função motora residual destes pacientes. A flexibilidade da região lombo sacra e da musculatura posterior de coxa utiliza-se o teste de sentar e alcançar no banco de Wells. ${ }^{2}$ Para aptidão muscular o teste de 10 repetições máximas e o teste de repetições máximas com Escala de Borg. ${ }^{1,3} \mathrm{Na}$ aptidão cardiorrespiratória aplica-se o teste caminhada de 6 minutos, caso o paciente for cadeirante utiliza-se o teste de 12 minutos impulsionando a cadeira de rodas, apenas com pacientes de risco baixo para doenças cardiovasculares, teste de esforço realizado pelo serviço de cardiologia no laboratório..$^{1,4,5}$

Composição corporal é usada o IMC; ${ }^{2} \mathrm{Na}$ avalição funcional é verificada a capacidade funcional de sentar e levantar da cadeira no teste de cinco repetições ou teste de 30 segundos. ${ }^{6}$ $\mathrm{O}$ equilíbrio dinâmico é avaliado no teste de levantar e caminhar (Time Up and GO). ${ }^{\text {? }}$

\section{Exercício físico}

O exercício físico no programa de reabilitação visa fazer com que os pacientes atinjam benefícios fisiológicos e psicológicos através de atividades com riscos controlados. Fundamentado nas diretrizes do American College of Sports Medicine (ACSM) construiuse o programa de condicionamento Físico combinado, que incluem treinamento aeróbio, de resistência muscular e flexibilidade articular. Este tipo de programa bem elaborado promove adaptações cardiovasculares, aumenta a força muscular, reduz gordura corporal e eleva a taxa metabólica basal. ${ }^{1}$

\section{Intervenção com exercício aeróbio}

A prescrição do programa de exercícios aeróbios é realizada de forma individualizada, a partir da avaliação médica cardiológica, para aqueles que foram ou não submetidos a teste de esforço.

As adaptações fisiológicas induzidas pelo treinamento aeróbio dependem principalmente da intensidade de sobrecarga. Existem várias maneiras diferentes de prescrever e enunciar a intensidade do 
exercício, neste protocolo utiliza-se três formas diferentes, o percentual da frequência cardíaca máxima $\left(\mathrm{FC}_{\text {Máx }}\right)$ obtido por meio do teste ergométrico ou ergoespirométrico, frequência cardíaca máxima $\left(\mathrm{FC}_{\text {Máx }}\right)$ obtida pela idade ou a Taxação de Esforço Percebido (TEP). ${ }^{1}$

A Intensidade inicial de treinamento aeróbio leva em consideração o nível inicial de aptidão aeróbia do paciente, é prescrita de leve a moderada, ou seja, a frequência cardíaca de treino (FCt) é de $60 \%$ a $80 \%$ da FC Max' obtida no teste de ergométrico utilizando formula de Karvonen para o cálculo. ${ }^{8}$

$\mathrm{Na}$ falta do teste de esforço, a intensidade do exercício pode ser controlada pela FCt de $60 \%$ a $80 \%$ FC Max obtida pela idade na fórmula de Tanaka. ${ }^{5}$ Exceto para pacientes em uso de betabloqueadores e/ou inibidores de canais de cálcio não-diidropiridinicos. ${ }^{5}$ Para estes pacientes não submetidos ao teste de esforço utiliza-se a Taxação de Esforço Percebido (TEP) 11 - 13 na Escala de Borg.

A Taxação de Esforço Percebido (TEP) pela Escala Subjetiva de Borg é uma abordagem psicofisiológica, onde o indivíduo que está se exercitando classifica numa escala numérica as sensações percebidas relativas ao nível de esforço. O aprimoramento e o ajuste da TEP durante o exercício proporcionam um meio efetivo de prescrever o exercício aeróbio sem ser a partir da frequência cardíaca máxima obtido no teste de esforço ou pela idade e sim a partir da percepção do esforço pelo indivíduo que coincide com as medidas objetivas da sobrecarga fisiológica/ metabólica como o \% da FCmax. \% do VO2 max. e concentração de lactato. ${ }^{10}$

A frequência do treinamento aeróbio pode ser de duas a três vezes por semana, com duração de 20 a 30 minutos. ${ }^{1}$ Os tipos de exercícios mais utilizados são pedalagem em cicloergômetro de MMII ou MMSS, caminhada com ou sem obstáculos (treino neuromuscular), treinamento intervalado, transporte sobre rodas, movimentos contínuos de extensão e flexão de ombros com extensão e flexão de cotovelos e braços alternados com tensores elásticos. ${ }^{3} \mathrm{O}$ educador físico deverá manipular a carga do equipamento utilizado e/ou sua velocidade. O ritmo da caminhada também deverá ser controlado a fim de o paciente realizar seu treino aeróbio dentro da FCt mínima e máxima prescrita.

O controle da FCt é realizada através da palpação do pulso radial a cada 10 minutos, para pacientes sem FCt verifica-se e registra a FC e a TEP pela Escala de Borg a cada 10 minutos, para controle da intensidade do treino aeróbio.
Após o término do exercício aeróbio, aguardar a recuperação da FC do paciente impondo intensidade mais baixa ou apenas sentado, por no mínimo dois minutos e no máximo cinco minutos e fazer nova aferição.

Habitualmente pacientes com aptidão aeróbia inicial muito baixa, nas primeiras sessões de exercício aeróbio, sempre sofrerão de fadiga periférica seja em MMII ou MMSS, antes que ocorra a fadiga central esperada, portanto, o exercício aeróbio deverá progredir de sessões curtas de 5 a 10 minutos de intensidade baixa e/ou moderada, alternada com períodos ativos de recuperação, ou seja, o exercício aeróbio de forma intervalada. ${ }^{3}$

\section{Intervenção com exercício resistido}

A efetividade de um programa de treinamento resistido depende de alguns fatores como intensidade, volume de treinamento, e frequência. Ao desenvolver o protocolo de prescrição de treino resistido, considerou-se o que constitui um equilíbrio ótimo dessas variáveis para maximizar os benefícios, sempre levando em consideração necessidades individuais como, tipo de sequela motora, idade, estado de saúde, nível de condicionamento, razões para o treinamento e metas pessoais.

Para aprimorar a força e a resistência muscular, fundamentou-se na intensidade de exercício resistido que permita ao indivíduo completar 8 a 12 repetições por série realizada de forma confortável. Isso equivale à intensidade moderada de $60 \%$ a $75 \%$ de uma repetição máxima. Com volume de treino no qual cada um dos principais grupos musculares deve ser treinado uma, duas ou três series com número de oito a dez exercícios, para pacientes que deambulam e para cadeirantes de cinco a oito exercícios por sessão de condicionamento físico. ${ }^{4,11}$

Frequência de duas a três vezes por semana com no mínimo 48 horas de intervalo entre as sessões de treinamento. A progressão de carga deve ser feita lentamente enquanto o paciente se adapta ao programa de $1 \mathrm{~kg}$ a 2 kg para MMSS e $2 \mathrm{~kg}$ a 4,5kg para MMII. Após esse período a progressão deve aumentar as cargas com acréscimo de $5 \%$ quando o paciente conseguir fazer 12 a 15 repetições de forma confortável ou quando o paciente referir uma TEP abaixo de 11 na Escala de Borg durante um exercício. ${ }^{3}$

Pacientes que não possuem boa força de preensão podem utilizar tensores elásticos, faixas elásticas ou cintos com velcro para prender suas mãos aos halteres ou ao equipamento com peso. ${ }^{4} \mathrm{Em}$ Lesados Medulares dar atenção especial ao desequilíbrio muscular e à prevenção das lesões por sobrecarga repetitiva. Os músculos antagonistas da propulsão em cadeira de rodas devem ser fortalecidos e os agonistas enfatizar o alongamento. A estabilização do tronco deve ser feita por cintos ou faixas, se necessário prender as mãos nos aparelhos ou halteres para que o paciente não tenha seu desempenho reduzido. Ressaltar o fortalecimento dos estabilizadores do ombro. ${ }^{3}$

Para Pacientes Hemiplégicos sempre que possível exercitar o membro superior hemiplégico/hemiparetico, pois o exercício é muito eficaz no tratamento da espasticidade, por promover a neuroplasticidade e melhora da elasticidade nos tecidos musculares e conjuntivo.

Ombro doloroso é frequente em pacientes com hemiplegia/hemiparesia, portanto muito cuidado ao prescrever exercícios para este grupo muscular. Evitar abdução horizontal desde ombro maior que 60ㅇ e evitar realizar exercícios na presença da dor. Com frequência estes pacientes, durante as séries, projetarão seu corpo na direção do lado mais fraco a fim de compensar esta fraqueza. Certifique-se que o paciente realizará a técnica apropriada. ${ }^{4}$

Em pacientes amputados dar ênfase a musculatura de tronco, glúteos e parte superior da coxa, por ser esta região uma das mais importantes para deambulação nesta população, que necessita de ganho de força e amplitude de movimento. ${ }^{3} \mathrm{Em}$ pacientes com déficit de coordenação dar preferência aos exercícios em máquinas, pois o movimento pode ser controlado oferecendo menos risco ao indivíduo.

\section{Recomendações e cuidados}

O esforço físico moderado comporta um pequeno risco de morte súbita durante a atividade, porém esse risco declinou muito nos últimos 25 anos, mesmo com o aumento do número de pessoas que começaram a praticar exercício físico.

Para diminuir a probabilidade de uma intercorrência relacionada ao exercício, algumas condições especiais devem ser observadas mais de perto no ambiente clinico. ${ }^{1}$

Antes de iniciar a sessão de condicionamento físico, é importante obter informações acerca do estado físico e emocional do indivíduo como, se fez uso de suas medicações adequadamente, se sente 
bem ou cansado naquele momento, se há algo a relatar referente à sessão anterior como dores musculares ou fadiga excessiva e também, seu estado de saúde (Quadro 1). 0 desempenho durante a sessão de exercícios está totalmente relacionado a esses fatores. ${ }^{12}$

A Sociedade Brasileira de Cardiologia Hipertensão e Nefrologia recomenda que, em indivíduos hipertensos a sessão de treinamento não deve ser iniciada se a pressão arterial sistólica (PAS) e a pressão arterial diastólica (PAD) estiverem superiores a 160 e/ ou $105 \mathrm{mmHg}$ respectivamente. ${ }^{5}$

Para este protocolo, o serviço de cardiologia da instituição percebeu a necessidade em reduzir esses parâmetros pressóricos para $150 \mathrm{mmHg}$ para PAS e $100 \mathrm{mmHg}$ para PAD para pacientes com histórico de eventos cardiorrespiratórios ou cardiovasculares, além da elaboração de recomendações na tomada de decisão para quando realizar ou não a sessão de condicionamento físico (Quadro 2) ${ }^{12}$

O Quadro 3 apresenta algumas recomendações para a interrupção da sessão, caso o paciente apresente certos sinais ou sintomas que exijam uma avaliação da equipe de enfermagem e/ou médica. ${ }^{12}$

Os pacientes diabéticos devem ter a glicemia monitorada antes, durante e após o exercício. Os níveis de glicemia abaixo de $100 \mathrm{mg} / \mathrm{dl}$ e acima de $250 \mathrm{mg} / \mathrm{dl}$ contra indica a prática de exercícios físicos. Outras recomendações também são necessárias, conforme descritas no Quadro $2 .{ }^{12}$

Pacientes com lesões da medula espinhal de T1-T6 experimentam disreflexia autônoma, termorregulação precária e hipotensão ortostática. A capacidade respiratória é reduzida ainda mais em virtude da paralisia dos músculos intercostais.

Durante o exercício, a disreflexia autônoma resulta em maior liberação de catecolaminas que irá aumentar a FC, o VO2, a pressão arterial (PA) e capacidade do indivíduo exercitar-se. Em algumas circunstancias, a PA pode estar elevada até níveis suficientemente

Quadro 1. Recomendações ${ }^{5}$ na tomada de decisão acerca da conveniência em realizar ou não sessão de Condicionamento físico

\begin{tabular}{|c|c|c|}
\hline \multicolumn{3}{|l|}{ Hipertensão assintomática } \\
\hline igual ou acima $150 \times 100 \mathrm{mmHg}$ & $\begin{array}{l}\text { Pacientes com antecedentes } \\
\text { de doenças cardiorrespiratória, } \\
\text { cardiovascular e vasculopatia; }\end{array}$ & $\begin{array}{c}\text { Dispensar o paciente da sessão } \\
\text { neste dia; }\end{array}$ \\
\hline \multicolumn{3}{|l|}{ Hipertensão sintomática } \\
\hline igual ou acima $150 \times 100 \mathrm{mmHg}$ & $\begin{array}{l}\text { Pacientes com antecedentes } \\
\text { de doenças cardiorrespiratória, } \\
\text { cardiovascular e vasculopatia; }\end{array}$ & $\begin{array}{c}\text { Dispensar o paciente da sessão } \\
\text { e encaminhar a equipe de } \\
\text { enfermagem; }\end{array}$ \\
\hline \multicolumn{3}{|l|}{ Hipertensão assintomática } \\
\hline igual ou acima $160 \times 100 \mathrm{mmHg}$ & $\begin{array}{l}\text { Pacientes sem antecedentes } \\
\text { de doenças cardiorrespiratória, } \\
\text { cardiovascular e vasculopatia; }\end{array}$ & $\begin{array}{c}\text { Dispensar o paciente se após } 15 \\
\text { minutos de exercício aeróbio leve a } \\
\text { PA se manter ou elevar; }\end{array}$ \\
\hline \multicolumn{3}{|l|}{ Hipertensão sintomática } \\
\hline igual ou acima $160 \times 100 \mathrm{mmHg}$ & $\begin{array}{l}\text { Pacientes sem antecedentes } \\
\text { de doenças cardiorrespiratória, } \\
\text { cardiovascular e vasculopatia; }\end{array}$ & $\begin{array}{c}\text { Dispensar o paciente da sessão } \\
\text { e encaminhar a equipe de } \\
\text { enfermagem; }\end{array}$ \\
\hline \multicolumn{3}{|l|}{ Hipertensão assintomática } \\
\hline igual ou acima $150 \times 95 \mathrm{mmHg}$ & $\begin{array}{l}\text { Pacientes com antecedentes } \\
\text { de doenças cardiorrespiratória, } \\
\text { cardiovascular e vasculopatia; }\end{array}$ & $\begin{array}{c}\text { Dispensar o paciente se após } 15 \\
\text { minutos de exercício aeróbio leve a } \\
\text { PA se manter ou elevar; }\end{array}$ \\
\hline
\end{tabular}

Quadro 2. Recomendações ${ }^{13}$ e cuidados no exercício para pacientes diabéticos

\begin{tabular}{l} 
- Não aplique insulina na região que será muito solicitada durante o exercício; \\
- $\quad$ Ajustar a dose e o tipo de insulina sob orientação médica, para realização do exercício; \\
- $\quad$ Ter sempre uma fonte de carboidratos para uso imediato, durante ou após o exercício; \\
- $\quad$ Ao primeiro sinal de hipoglicemia, interromper o exercício e ingerir carboidratos; \\
Cuidados no exercício para diabéticos em presença de complicações crônicas: \\
- $\quad$ Retinopatia: evitar exercícios que elevem muito a pressão arterial sistólica ou que exijam manobra de valsalva; \\
- Nefropatia: evitar exercício de alta intensidade, devido ao aumento da proteinúria; \\
\hline - Neuropatia periférica: usar palmilhas de silicone, meias de algodão sem costura, examinar os pés após exercício e, em casos mais graves \\
- $\quad$ Neuropatia autonômica: evitar exercícios que obriguem a mudança brusca de posição e exercícios em temperaturas extremas;
\end{tabular}

altos para produzir um acidente vascular encefálico (PAS $250-300 \mathrm{mmHg}$ ou PAD de 200-220 mmHg). ${ }^{3}$ Por esta razão é necessário aferir a pressão arterial antes do início da sessão com exercícios físicos.

As recomendações e os cuidados quanto a disreflexia estão descritas no Quadro 3 e inclui um cálculo para determinar a PA média destes pacientes.

\section{CONCLUSÃO}

A participação em um programa de condicionamento físico na reabilitação de pessoas acometidas por distúrbios neuromusculares e musculoesqueléticos tem a atenção focada na compreensão dos mecanismos pelos os quais o exercício aprimora a saúde, a aptidão física e o potencial de reabilitação. Este protocolo elucida várias aplicações clinicas da intervenção com exercícios físicos que podem influenciar positivamente na saúde do paciente.

No ambiente clínico, o educador físico deve atuar de maneira a proporcionar melhora no condicionamento físico global do paciente, principalmente no que se refere a força muscular, condicionamento cardiorrespiratório e equilíbrio, oportunizando assim melhor qualidade de vida e autonomia a esses indivíduos.

\section{REFERÊNCIAS}

1. Mcardle WD. Fisiologia do exercício: energia, nutrição e desempenho humano. Rio de Janeiro: Guanabara Koogan; 2008.

2. American College Sports of Medicine. Manual do ACMS para avaliação da aptidão física relacionada à saúde. Rio de Janeiro: Guanabara Koggan; 2006.

3. American College of Sports Medicine. Diretrizes do ACSM para os testes de esforço e sua prescrição. 8 ed. Rio de Janeiro: Guanabara Koogan; 2010.

4. American College of Sports Medicine. Pesquisas do ACSM para a fisiologia do exercício clínico: afecções musculoesqueléticas, neuromusculares, neoplásicas, imunológicas e hematológicas. Rio de Janeiro: Guanabara Koogan; 2004.

5. Sociedade Brasileira de Cardiologia / Sociedade Brasileira de Hipertensão / Sociedade Brasileira de Nefrologia. VI Diretrizes Brasileiras de Hipertensão. Arq Bras Cardiol. 2010; 95(1 supl.1):1-51. DOI: https://doi.org/10.1590/S0066782X2010001700001

6. Jones CJ, Rikli RE, Beam WC. A 30-s chair-stand test as a measure of lower body strength in communityresiding older adults. Res Q Exerc Sport. 1999 Jun;70(2):113-9. DOI: https://doi.org/10.1080/0270 1367.1999.10608028

7. Podsiadlo D, Richardson S. The timed "Up \& Go": a test of basic functional mobility for frail elderly persons. Am Geriatr Soc. 1991;39(2):142-8. DOI: https://doi. org/10.1111/j.1532-5415.1991.tb01616.x 
Quadro 3. Recomendações5 e cuidados no exercício para pacientes com lesão medular Recomendações

Para pacientes com lesão medular acima do nível T6, monitorar PA regularmente (início, meio e fim), do exercício aeróbio e exercício resistido; PA de repouso do paciente deve ser inferior ou igual a sua PA média, para ser liberado para iniciar a sessão de CF.

\section{PA media é identificada da seguinte forma:}

Verificar a PA de repouso nas três primeiras sessões de CF; Somar os resultados obtidos de PAS e dividir pelo número de resultados; Somar os resultados de PAD e dividir pelo número de resultados; O valor obtido nesta equação será a PA média do paciente.

\section{Ações e desvios caso paciente se apresente com alteração de PA}

\section{Caso paciente se apresente ao serviço:}

Com suspeita de disreflexia: somar $20 \mathrm{mmHg}$ a PA media do paciente, se a PA de repouso estiver menor que PA média $+20 \mathrm{mmHg}$ deve-se prosseguir a sessão normalmente; se maior ou igual a PA media $+20 \mathrm{mmHg}$, dispensar o paciente e encaminhá-lo ao serviço de enfermagem para avaliação.

Sem suspeita de disreflexia: somar $30 \mathrm{mmHg}$ a PA media do paciente, se a PA de repouso estiver menor que PA média $+30 \mathrm{mmHg}$ deve-se prosseguir a sessão normalmente, se maior ou igual a PA media $+30 \mathrm{mmHg}$, dispensar o paciente e encaminhá-lo ao serviço de enfermagem para avaliação.

Tetraplégicos mesmo assintomáticos, se PA sistólica maior ou igual a 140 e/ou PA diastólica maior ou igual a 90 , suspender a terapia e encaminhar ao posto de enfermagem para avaliação médica e, pacientes lesados medulares com paraplegia mesmo assintomáticos, se PA sistólica maior ou igual a 150 e/ou PA diastólica maior ou igual a 100, suspender a terapia e encaminhar ao posto de enfermagem para avaliação médica.

Em caso de aumento da PAS e PAD, com os sintomas como bradicardia, sudorese, cefaleia latejante, rubor facial e congestão nasal deve-se sentá-lo com coluna na vertical para reduzir a PA e identificar e remover o estimulo irritante. Se necessário, interromper o exercício imediatamente, encaminhar o paciente para o serviço de enfermagem.

\section{Cuidados}

O exercício aeróbio deverá progredir de sessões curtas de 5 a 10 minutos de intensidade moderada, alternada com períodos ativos de recuperação com duração de 5 minutos.

Caso o paciente apresente um quadro de hipotensão ortostática induzida pelo exercício: reposicionar sua cadeira para uma posição inclinada (cabeça mais baixa que os pés), ou deitar o paciente com elevação das pernas; se não houver melhora, encaminhar ao posto de enfermagem para avaliação médica. Em pacientes cadeirantes enfatizar alongamento dos músculos agonistas da propulsão em cadeira de rodas (músculos do ombro anterior e peitoral) e os antagonistas devem ser fortalecidos (músculos do ombro posterior e parte superior das costas);

Evitar fatores que reduzem a tolerância ao calor como perda de sono, álcool, doença infecciosa, desidratação e falta de aclimatação.
8. Karvonen MJ, Kentala E, Mustala O. The effects of training on heart rate; a longitudinal study. Ann Med Exp Biol Fenn. 1957;35(3):307-15.

9. Tanaka H, Monahan KD, Seals DR. Age-predicted maximal heart rate revisited. J Am Coll Cardiol. 2001;37(1):153-6. DOI: https://doi.org/10.1016/ S0735-1097(00)01054-8

10. Borg GA. Psychophysical bases of perceived exertion. Med Sci Sports Exerc. 1982;14(5):377-81. DOI: https:// doi.org/10.1249/00005768-198205000-00012

11. Pollock ML, Franklin BA, Balady GJ, Chaitman BL, Fleg $\mathrm{JL}$, Fletcher $\mathrm{B}$, et al. Resistance exercise in individuals with and without cardiovascular disease: benefits, rationale, safety, and prescription: An advisory from the Committee on Exercise, Rehabilitation, and Prevention, Council on Clinical Cardiology, American Heart Association; Position paper endorsed by the American College of Sports Medicine. Circulation. 2000;101(7):828-33. DOI: https://doi.org/10.1161/01. CIR.101.7.828

12. Yazbek Junior P, Sabbag LMS, Battistella LR. Tratado de reabilitação: diretrizes nas afecções cardiovasculares, neuromusculares e musculoesqueléticas. São Paulo: Phorte; 2010.

13. Diretrizes da Sociedade Brasileira de Diabetes (20152016). São Paulo: A.C. Farmacêutica; 2016. 